\title{
Visible Light Tomography Considering Reflection Light in a Small Tokamak Device PHiX ${ }^{*}$
}

\author{
Koyo MUNECHIKA, Hiroaki TSUTSUI and Shunji TSUJI-IIO \\ Tokyo Institute of Technology, Tokyo 152-8550, Japan
}

(Received 30 November 2020 / Accepted 1 February 2021)

\begin{abstract}
In a small tokamak, the visible light emission is observed and used to investigate plasmas' behavior with a fast visible camera. However, the reflected light causes a systemic error in measuring visible light emitted from the plasma. In this paper, we managed to overcome the reflection effect with the ray-tracing technique which is utilized in a synthetic diagnostic platform of the small tokamak device PHiX at Tokyo Institute of Technology using Raysect and CHERAB python libraries. We successfully evaluated the amount of reflected light and obtained tomographic reconstruction images from simulated and experimental data with the TikhonovPhillips regularization and the L-curve method to choose an optimal regularization parameter. We also proposed to project the contour of a reconstruction image onto a camera image to validate tomography results.
\end{abstract}

(c) 2021 The Japan Society of Plasma Science and Nuclear Fusion Research

Keywords: high-speed camera, tomographic reconstruction, visible light, reflection light, Tikhonov-Phillips, Lcurve, ray tracing, small tokamak, synthetic diagnostics platform

DOI: $10.1585 /$ pfr. 16.2402033

\section{Introduction}

Recently, the research and development of DEMO fusion reactors have been conducted in the world [1,2], the type of which is the tokamak device with high plasma confinement performance. Tokamaks have H-mode discharge states with sufficient energy confinement [3]. To maintain this state and achieve an economical steady-state fusion operation, it is essential to control neutral and impurity particle behavior and understand their spatial distribution [3]. Notably, the investigation of visible light emission around the edge region of plasma enables us to determine the mechanism of transport of neutral and impurity particles. One of the ways to tackle these issues is a tomographic reconstruction technique using a high-speed camera, which can determine the spatial distribution of visible light emission.

High-speed visible camera measurements are widely used as essential equipment for plasma experiments [4]. Using a tangentially viewing camera allows us to understand the plasma boundary shape, position, fluctuations, etc. The tomographic reconstruction technique is also used to evaluate the poloidal cross-section distribution of visible light emitted from the plasma [5]. However, the reflection light causes crucial artifacts in reconstructed images. The previous study addressed reflection effects using two models: Phong and Lambert models, which are associated with specular and diffusive effects, respectively [6]. They assumed that the tokamak inner wall was axisymmetric while the mask images were superimposed on the

author'se-mail: munechika.koyo@torus.nr.titech.ac.jp

*) This article is based on the presentation at the 29th International Toki Conference on Plasma and Fusion Research (ITC29). wall to demonstrate nonaxisymmetric components. Besides, only the first reflection on the wall surface was taken into account. In a small tokamak, however, the vessel wall consists of almost mirror surfaces, and it has a lot of nonaxisymmetric components, so we need to consider a more realistic environment with a ray-tracing technique that enables us to generate images based on geometric optics.

In this paper, we used the Phantom LAB110 highspeed camera equipped with a small tokamak device PHiX [7] at Tokyo Institute of Technology. Main parameters of PHiX and the camera are shown in Tables 1 and 2, respectively. First, to simulate camera images and evaluate the amount of reflected light, we developed the synthetic diagnostic platform with Raysect [8] and CHERAB [9], offering the ray tracer and calculations for simulated emission data. We then produced reconstructed images using camera images calculated by Raysect and experimental data captured by the high-speed camera (See Sec. 4.1). We also facilitated comparing the reconstructed image with the corresponding camera image by projecting the reconstructed image's contour onto the camera image using the calcam library [10] (See Sec. 4.2).

\section{Development of Synthetic Diagnostics Platform}

Creating a virtual model by theoretical calculations and measurements with a virtual instrument on a computer is called synthetic diagnostics. The virtual experiment environment platform, including them, is called the synthetic diagnostics platform [11]. The synthetic diagnostics enables us to compare theoretical and experimental values, which is used to identify parameters that cannot be directly 
Table 1 Main parameters of PHiX.

\begin{tabular}{cc}
\hline \hline Quantity & Value \\
\hline Major radius $R_{\mathrm{p}}$ & $33 \mathrm{~cm}$ \\
Minor radius $a_{\mathrm{p}}$ & $9 \mathrm{~cm}$ \\
Plasma current $I_{\mathrm{p}}$ & $<5 \mathrm{kA}$ \\
Toroidal magnetic field $B_{\mathrm{t}}$ & $<0.3 \mathrm{~T}$ \\
Elongation $\kappa$ & $<1.5$ \\
Pulse duration $\tau_{\text {pulse }}$ & $12 \mathrm{~ms}$ \\
\hline \hline
\end{tabular}

Table 2 Main parameters of the high-speed camera.

\begin{tabular}{cc}
\hline \hline Quantity & Value \\
\hline frame rate & $10000 \mathrm{fps}$ \\
exposure time & $99 \mu \mathrm{s}$ \\
pixel resolution (width, high) & $(256,512) \mathrm{px}$ \\
pixel pitch & $20 \mu \mathrm{m}$ \\
focal length & $10 \mathrm{~mm}$ \\
working distance & $50 \mathrm{~cm}$ \\
F value & 3.5 \\
\hline \hline
\end{tabular}
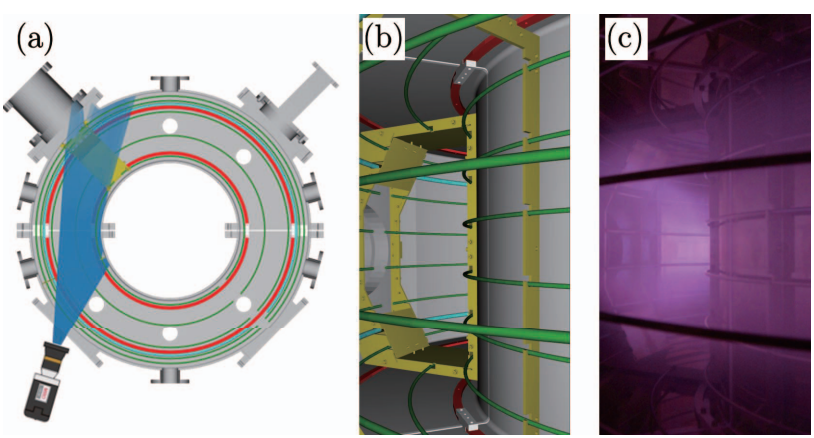

Fig. 1 (a): Top-down view of PHiX with camera's field of view (in blue area). (b): Field of view in CAD space. The inner vessel components are colored (e.g. flux loop: green, limiter: yellow, rail supporting them: red). (c): Captured image in shot No.17393.

measured in the experiment and verify a theory. In this study, we theoretically created an emission distribution in PHiX and a camera image by ray tracing. Their results were used not only as a comparison with actual camera images but also as given data for verifying tomography. Figure 1 shows the components (vessel, flux loops, in-vessel coils, etc.) and field of view of a high-speed camera, which are taken into account in the development of a synthetic diagnostics platform.

Raysect, which is a ray-tracing framework in Python [8], can deal with the physical light effect such as reflection, refraction, scattering, etc.

A high-speed camera captures the emission arriving at each pixel through the lens. To compute the radiant flux $\Phi_{1 \mathrm{px}}$ at a pixel with its surface area $A_{1 \mathrm{px}}$, we applied the thin lens model as:

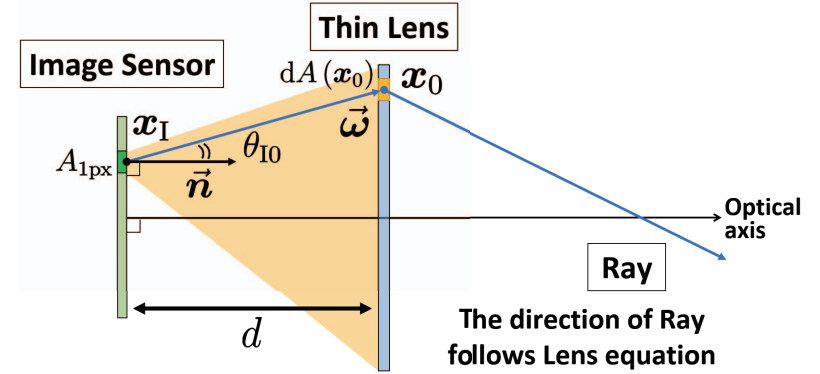

Fig. 2 Schematic diagram of the thin lens model.

$$
\begin{aligned}
\Phi_{1 \mathrm{px}}= & \int_{0}^{\infty} \mathrm{d} \lambda \int_{A_{\mathrm{lpx}}} \mathrm{d} A\left(\boldsymbol{x}_{\mathrm{I}}\right) \int_{A_{\mathrm{lens}}} \mathrm{d} A\left(x_{0}\right) \\
& \times L\left(\boldsymbol{x}_{0} \rightarrow \boldsymbol{x}_{\mathrm{I}}, \lambda\right) \frac{\cos ^{4} \theta_{0 \mathrm{I}}}{d^{2}} .
\end{aligned}
$$

Here, $\boldsymbol{x}_{\mathrm{I}}$ and $\boldsymbol{x}_{0}$ represent the positions in a pixel and the passing point in the lens, respectively. $A_{\text {lens }}$ is the area of lens. The incident radiance $L\left(\boldsymbol{x}_{0} \rightarrow \boldsymbol{x}_{\mathrm{I}}, \lambda\right)$ with the wavelength $\lambda$ is computed by the rendering equation [12]. $\theta_{0 \mathrm{I}}$ is the angle between $\boldsymbol{x}_{0}-\boldsymbol{x}_{\mathrm{I}}$ and normal vector $\overrightarrow{\boldsymbol{n}}$ to the pixel, and $d$ is the distance between image sensor and thin lens. See Fig. 2 for a schematic representation of this model.

The radiance $L\left(x_{0} \rightarrow x_{\mathrm{I}}, \lambda\right)$ is equal to $L\left(x_{1} \rightarrow x_{0}, \lambda\right)$, where $x_{1}$ is the first point which the ray hits after launched from the lens point $\boldsymbol{x}_{0} . \quad L\left(\boldsymbol{x}_{1} \rightarrow \boldsymbol{x}_{0}, \lambda\right)$ is composed by the volume emission and the wall reflection term as the following equation which is referred to as the rendering equation:

$$
\begin{aligned}
& L\left(x_{1} \rightarrow \boldsymbol{x}_{0}, \lambda\right)=\int_{\boldsymbol{x}_{0}}^{\boldsymbol{x}_{1}} j(\boldsymbol{x}, \overrightarrow{\boldsymbol{\omega}}, \lambda) \mathrm{d} s \\
& \quad+\int_{\Omega} \mathrm{d} \omega_{\mathrm{i}} f_{\mathrm{r}}\left(\boldsymbol{x}_{1}, \overrightarrow{\boldsymbol{\omega}}_{\mathrm{i}}, \vec{\omega}, \lambda\right) L\left(\boldsymbol{x}_{2} \rightarrow \boldsymbol{x}_{1}, \lambda\right)\left|\cos \theta_{\mathrm{i}}\right| .
\end{aligned}
$$

The amount of incident radiance is given by the line integral of volumetric emission $j(\boldsymbol{x}, \vec{\omega}, \lambda)$ from $\boldsymbol{x}_{0}$ to the ray's first surface intersection point $\boldsymbol{x}_{1}$ and the integral of the incident radiance at the ray's intersecting surface over the collecting solid angle $\Omega$ which is contributed to reflection effects. The second integral term includes the bidirectional reflectance distribution function (BRDF) $f_{\mathrm{r}}\left(\boldsymbol{x}_{1}, \vec{\omega}_{\mathrm{i}}, \overrightarrow{\boldsymbol{\omega}}, \lambda\right)$ and $\cos \theta_{\mathrm{i}}=\overrightarrow{\boldsymbol{\omega}}_{\mathrm{i}} \cdot \overrightarrow{\boldsymbol{n}}$, where $\overrightarrow{\boldsymbol{\omega}}_{\mathrm{i}}$ and $\overrightarrow{\boldsymbol{n}}$ represent the incoming light direction and the normal vector to the ray's intersecting surface, respectively. $\boldsymbol{x}_{2}$ means the point which the ray hits secondly after the first reflection. The schematic diagram of this equation is shown in Fig. 3. The BRDF is a weighting function that describes the redistribution of incident light into outgoing reflections and transmission/absorption. Raysect implements the Cook-Torrance microfacet model as the BRDF [13], which enables us to change the characteristic of the material surface with only the roughness parameter, $a_{\mathrm{r}} \in[0,1]$. In this model, the material surface composes many microfacets, each of which is assumed to be the mirror-like surface and the Fresnel 


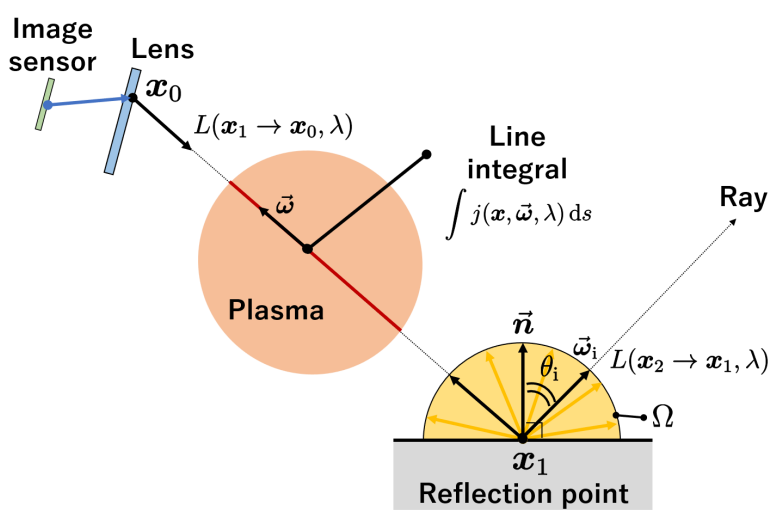

Fig. 3 Schematic diagram of the ray tracing method.

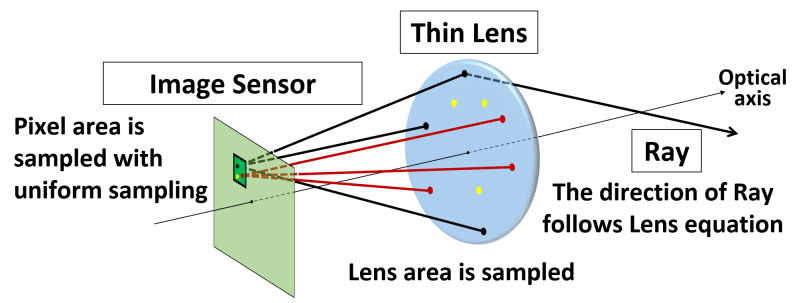

Fig. 4 Sampling positions of initiated rays on a pixel and those of rays passing through the thin lens.

reflection law where reflectivity is determined by the material refraction. As $a_{\mathrm{r}} \rightarrow 1$, the material surface becomes totally diffusive like an ideal Lambertian surface. In other hand, as $a_{\mathrm{r}} \rightarrow 0$, the bulk surface behaves specularly. See Fig. 3 in [12].

Raysect implements the Monte-Carlo method to solve these equations numerically and simulate camera images [12]. To consider the effect of multiple reflections, the direction of a reflected ray hitting the in-vessel components which are shown in Figs. 1 (a) (b) is determined statistically and the ray-tracing calculation is continued following the path tracing algorithm [12]. Figure 4 illustrates the sampling procedure of ray launching.

In this study, we decided the plasma shape and parameters using the Tokamak Simulation Code (TSC) [14] and experimental data measured by the Langmuir probe. Plasma electron density $n_{\mathrm{e}}$ and temperature $T_{\mathrm{e}}$ have been assumed to be quadratic as a function of normalized poloidal flux as:

$$
\begin{aligned}
& n_{\mathrm{e}}(\Psi)=\left(n_{\min }-n_{\max }\right) \Psi^{2}+n_{\min }, \\
& T_{\mathrm{e}}(\Psi)=\left(T_{\min }-T_{\max }\right) \Psi^{2}+T_{\min },
\end{aligned}
$$

where $\Psi$ is normalized poloidal flux whose value is unity at the magnetic axis and zero on the plasma surface, and $n_{\min }, n_{\max }$ and $T_{\min }, T_{\max }$ are the minimal and maximum electron density and temperature estimated by measurement, respectively. The hydrogen atom density is assumed to be a constant in space. As the visible light emission, we chose the $\mathrm{H}$-alpha line spectrum. Under these assump-

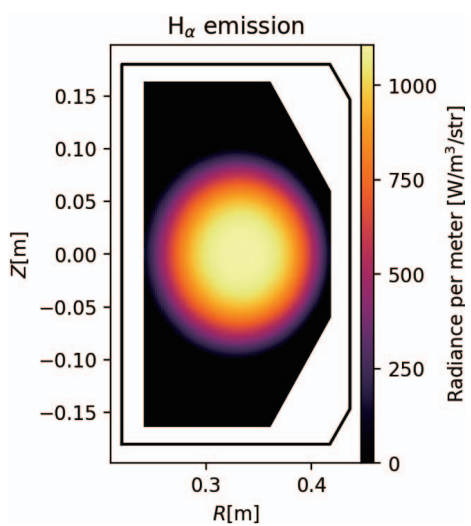

Fig. 5 Plasma emission $\left(\mathrm{H}_{\alpha}\right)$ data used in this work. The black wide line shows the outer edge of the limiter plate.

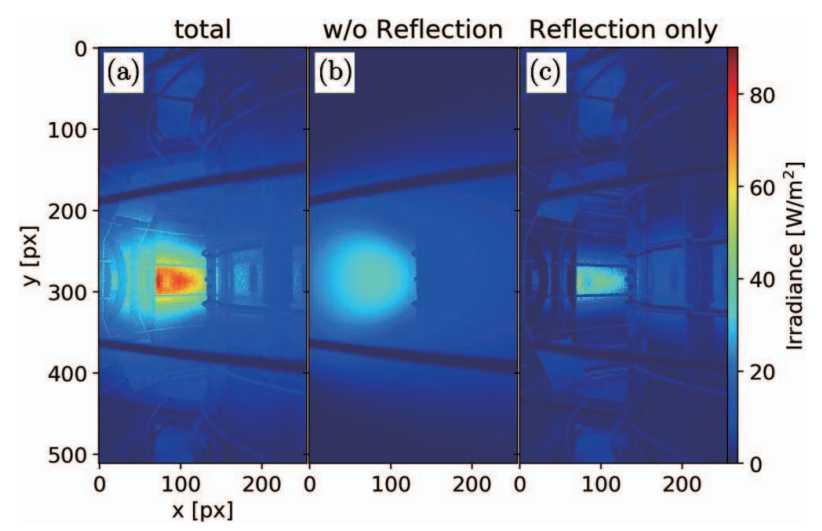

Fig. 6 (a): Total plasma simulated emission including reflection effects, (b): without reflection effects, and (c): the subtraction of images (a) and (b).

tions, the $\mathrm{H}$-alpha emission distribution was computed by CHERAB [9] (Fig. 5) in which $\mathrm{H}$-alpha emission is a function of $\mathrm{H}$-atom, and $\mathrm{H}$-ion and electron densities and temperatures. Then, we produced a camera simulation image with or without the reflection contribution (Fig. 6). Subtracting (b) from (a) in Fig. 6 enables us to evaluate reflected light's contribution. According to this, the amount of reflected emission was found to be almost equivalent to non-reflected emission in the brightest regions. This is why the reflected light needs to be considered if the visible light emission is dominant.

\section{Method of Tomographic Reconstruction}

What we have to do for tomography at first are to discretize the spatial distribution and to generate the grid space. In this work, axisymmetric plasma emission is assumed while reflections and in-vessel components are nonaxisymmetric. Using the assumption and Eqs. (1) and (2), we computed the Ray Transfer Matrix (RTM), also called a geometric matrix which relates the the volume emission of the plasma to the radiant flux on the camera pixel. The 
graphical representation of the cylindrical mesh and the contribution of a ray is shown in Fig. 7. In this work, the matrix consists of $32768 \times 13326$ elements where "32 768" and "13 326" are the number of camera pixels and the meshes on the $r-z$ plain, respectively. The values of RTM elements contributing to a camera pixel are plotted in the $r$ - $z$ plane (Fig. 8), which shows not only trajectories of the launched and reflected rays by the wall and nonaxisymmetric components like limiter, ports, etc. (shown

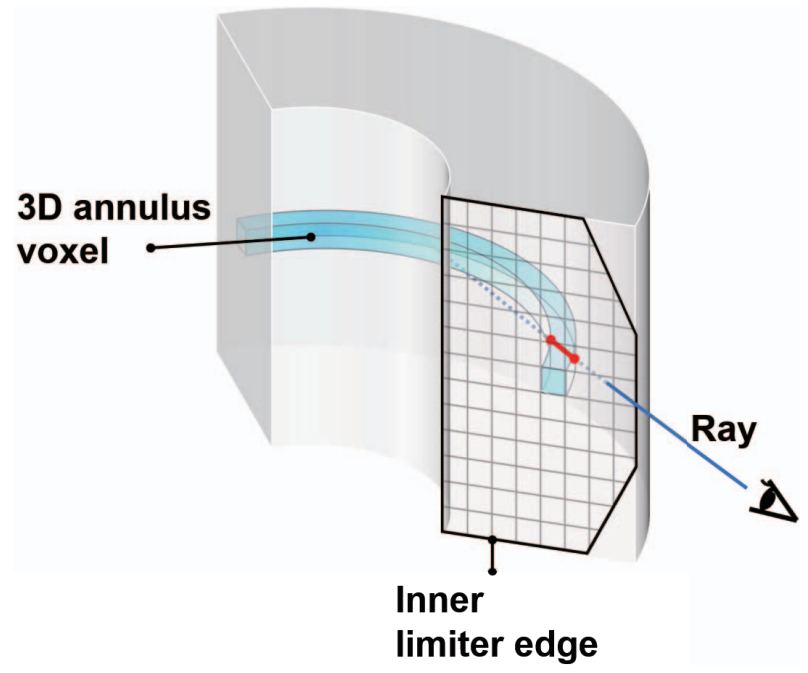

Fig. 7 Schematic diagram of the cylindrical mesh and the contribution of a ray. The calculation of ray position and interaction with in-vessel components is conducted in $(x, y, z)$ coordinates. On the other hand, each 3D voxel corresponding to the plasma emission is composed of a toroidally symmetric annulus described in $(r, z)$ coordinates. The length of the ray segment cut by the blue voxel is colored with red. This ray segment contributes to a certain RTM element corresponding to the voxel where the ray passes.

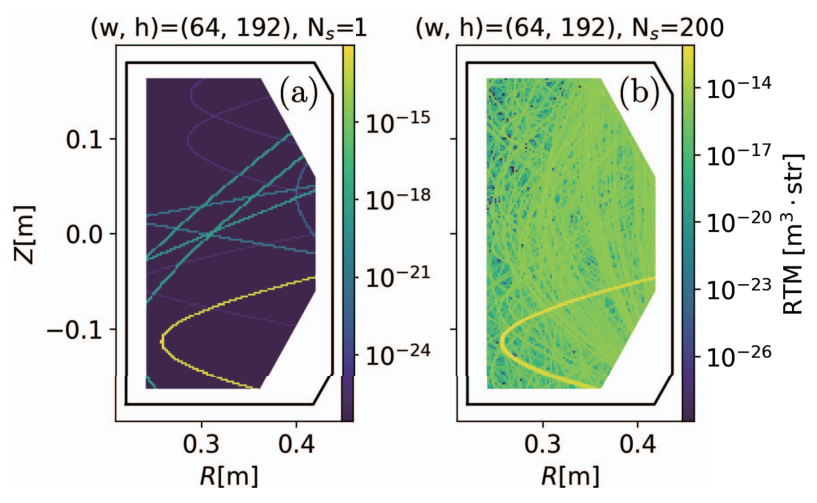

Fig. 8 The color maps of the RTM elements contributing to the image-sensor pixel at $(64,192)[p x]$ in log-scale. (a) The numbers of sampling points in a camera pixel and passing through the lens are 1 and 1 , respectively. (b) The numbers of those are 10 and 20, respectively. The number of total launched rays $N_{\mathrm{s}}$ is the product of the sampled numbers on the camera pixel and the lens. in Fig. 1) on the $r-z$ plain but also the values of RTM elements in the Monte-Carlo estimation.

Tomography is known as the ill-posed problem, so we need to estimate a reconstructed image vector $\boldsymbol{x}$ by solving the following equation:

$$
\begin{aligned}
& \boldsymbol{x}_{\lambda}=\arg \min \left\{\|A \boldsymbol{x}-\boldsymbol{b}\|^{2}+\lambda\|L \boldsymbol{x}\|^{2}\right\}, \\
& \boldsymbol{x}=0 \quad \text { (at the inner limiter edge), }
\end{aligned}
$$

where $\boldsymbol{x}_{\lambda}$ : estimated solution, $A$ : RTM, $\boldsymbol{b}$ : camera data, $\lambda$ : regularization parameter, $L$ : laplacian operator with the Dirichlet boundary condition $\boldsymbol{x}=0$ at the inner limiter edge (in Eq.6). The Dirichlet boundary condition can reduce the artifact emission by reflections just near the vacuum vessel. The estimation is based on the Tikhonov-Phillips regularization method [15]. And we applied the L-curve method to optimize the regularization parameter [16]. Notice that Tikhonov-Phillips regularization gives not only positive but also negative values in estimated solution.

\section{Results and Discussions 4.1 Simulated data}

To confirm the tomographic system, we retrieved a emission distribution from a simulated camera image (Fig. 6(a)) to which I added no noise. The relative error between the original distribution data $\boldsymbol{x}_{\mathrm{t}}$ and estimated solution $\boldsymbol{x}_{\lambda}$ is defined as follows:

$$
e(\lambda)=\frac{\left\|x_{\mathrm{t}}-\boldsymbol{x}_{\lambda}\right\|}{\left\|\boldsymbol{x}_{\mathrm{t}}\right\|} .
$$

From Fig. 9 (d), we found that the estimated solution by the L-curve method was very close to the true solution. Comparing the reconstructed image and true solution in Fig. 10, they are almost indistinguishable, and the optimized one seems to be smoothened around the cut edge $z= \pm 0.10$ m (Fig. 9(d)) due to larger regularization parameter $\lambda$. The fraction of the number of pixels containing a negative value
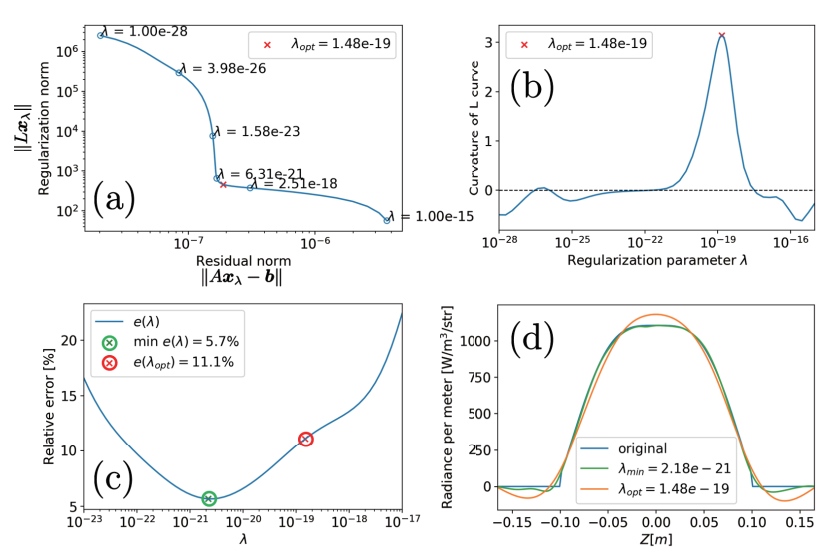

Fig. 9 (a): L-curve plot, (b): curvature of L-curve as a function of regularization parameter $\lambda$, (c): relative error as a function of $\lambda,(\mathrm{d})$ : reconstructed data at $r=0$. 

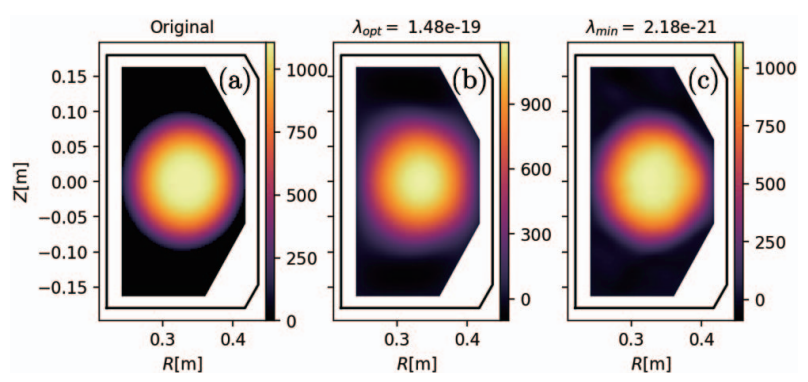

Fig. 10 (a): The model emission data as shown in Fig. 5, (b): the estimated reconstruction image with optimized regularization parameter $\lambda$ by the L-curve method, (c): the reconstructed image with $\lambda$ corresponding to the minimal relatively error.

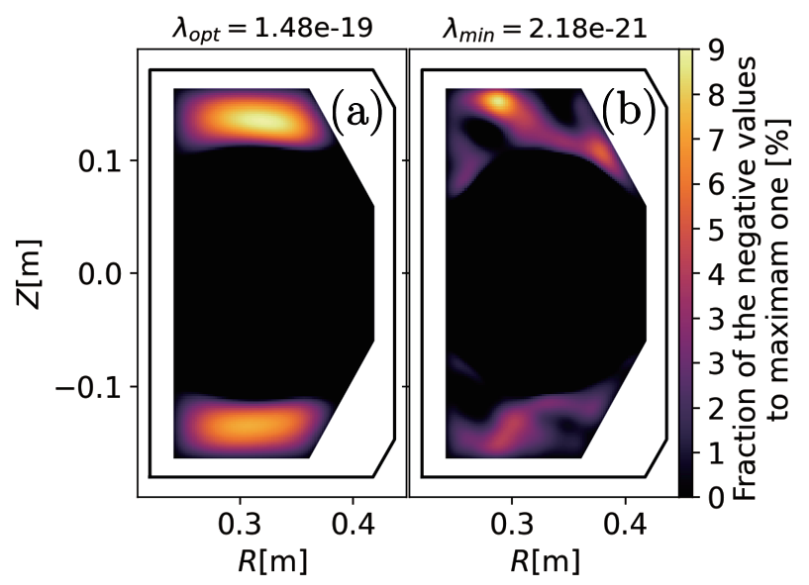

Fig. 11 The negative values of the reconstructed image (Fig. 10) normalized to the maximum reconstructed emission. (a) and (b) corresponds to (b) and (c) in Fig. 10, respectively.

in reconstructed image (Fig. 10 (b)) is about 26.3\%, and the distribution of them is shown in Fig. 11. This indicates that the negative values is much smaller than the positive ones.

\subsection{Experimental data}

We attempted to restore the cross section of visible light emission using a measured data of the shot number 17393. The discharge duration was about $5 \mathrm{~ms}$, and the plasma current reached up to $1.0 \mathrm{kA}$. Figure 12 shows the tomography results of some images captured with the high-speed camera during this discharge. Comparing camera images and the reconstructed images, it seems that the plasma's vertical movement during the shot is well represented, in which there is no reconstructed images having the negative value. The evaluation of reflection components in the RTM is effective, but it's not enough because the rightmost reconstructed image has two peaks and the outer one seems to be the effect of reflected light. Hence, we need to optimize the reflection parameter $a_{\mathrm{r}}$ to remove this error.

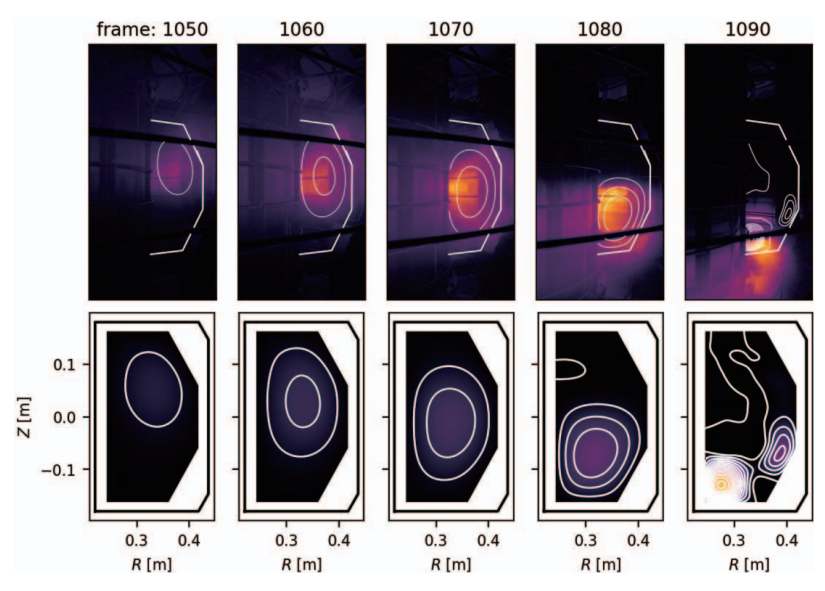

Fig. 12 Bottoms are reconstructed images and tops are camera frames which are flipped horizontally. The white contours representing the intensity of reconstruction are projected onto camera images to ease up on validating the tomography results. The inner edge of the limiter plate is also showed in camera images as straight white lines. This projection technique is based on the calcam library [10].

\section{Summary}

We developed a synthetic diagnostics platform for PHiX and successfully simulated the realistic camera images including reflection effects with the ray-tracing technique. The tomography results suggests that visible light emission distribution is restored in both simulation and experiment data. We found that we have to optimize the reflection parameter "roughness" to totally eliminate the artifact due to the wall effect.

\section{Acknowledgements}

This work is performed with the support and under the auspices of the NIFS Collaboration Research program (NIFS18KOAP033).

[1] G. Federici et al., Fusion Eng. Des. 136, 729 (2018).

[2] Y. Someya et al., Fusion Eng. Des. 136, 1306 (2018).

[3] I.P.B. Editors et al., Nucl. Fusion 39, 2137 (1999).

[4] H. Zhang et al., IEEE Trans. Plasma Sci. 46, 1312 (2018).

[5] J. Xiang et al., Fusion Eng. Des. 131, 166 (2018).

[6] M. Odstrčil et al., Rev. Sci. Instrum. 85, 013509 (2014).

[7] T. Kobayashi et al., in Proc. 42nd eps conf. plasma phys. (2015) P4.188.

[8] A. Meakins et al., raysect/source: v0.5.2 Release, 2018.

[9] D.C. Giroud et al., CHERAB Spectroscopy Modelling Framework, 2018.

[10] S. Silburn et al., Calcam, 2020.

[11] L. Shi et al., Rev. Sci. Instrum. 87, 11D303 (2016).

[12] M. Carr et al., Rev. Sci. Instrum. 90, 043504 (2019).

[13] B. Walter et al., Eurographics, 195 (2007).

[14] S.C. Jardin et al., J. Comput. Phys. 66, 481 (1986).

[15] N. Iwama et al., J. Plasma Fusion Res. 82, 399 (2006).

[16] P.C. Hansen, Comput. Inverse Probl. Electrocardiology, ed. P. Johnston, Adv. Comput. Bioeng. 4, 119 (2000). 